\title{
Avaliação nutricional seguida de ações educativas para promoção de hábitos alimentares saudáveis em mulheres com câncer de mama participantes de um grupo de apoio no sul de Minas Gerais
}

\author{
Nutrition evaluation followed by educational activities to promote healthy eating \\ habits in women with breast cancer attending a support group in southern \\ Minas Gerais
}

Geisa Oliveira Rocha ${ }^{1}$, Bruna Nogueira Andrade ${ }^{2}$, Edmar Ramos de Carvalho ${ }^{3}$, Rafaela Corrêa Pereira ${ }^{4}$, Michel Cardoso de Angelis-Pereira ${ }^{5}$, Glaucia Imaculada Soares Pereira ${ }^{6}$, Maria Camila Lima ${ }^{7}$

1 Estudante de graduação. Universidade Federal de Lavras (UFLA), Brasil. E-mail: geisa_or@hotmail.com

2 Estudante de graduação. Universidade Federal de Lavras (UFLA), Brasil. E-mail: bruna.nogueira.andrade@hotmail.com

3 Estudante de graduação. Universidade Federal de Lavras (UFLA), Brasil. E-mail: edmarlavras@hotmail.com

4 Doutoranda. Universidade Federal de Lavras (UFLA), Brasil. E-mail: rafacpereira@gmail.com

5 Professor Adjunto III. Universidade Federal de Lavras (UFLA), Brasil. E-mail: deangelis@dca.ufla.br

6 Nutricionista. Universidade Federal de Lavras (UFLA), Brasil. E-mail: gimspereira@lavras.mg.gov.br

7 Mestranda. Universidade Federal de Lavras (UFLA), Brasil. E-mail: mariacamilalima@yahoo.com.br

Recebido em: 31/03/2015 | Aprovado em: 18/11/2015

DOI: 10.12957/interag.2016.15487

\begin{abstract}
Resumo
Este trabalho teve como objetivo realizar avaliação nutricional em mulheres com câncer de mama que frequentavam um grupo de apoio para oferta de atividades de acolhimento, orientação e suporte terapêutico e, com base neste resultado, desenvolver ações de educação alimentar e nutricional (EAN). As atividades foram conduzidas por estudantes que cursavam a disciplina de EAN do curso de graduação em Nutrição da Universidade Federal de Lavras (UFLA). A qualidade da alimentação das participantes foi avaliada por meio da aplicação de questionário baseado no Guia Alimentar do Ministério da Saúde. Medidas antropométricas foram obtidas para o cálculo do índice de massa corporal (IMC) e da relação cinturaquadril (RCQ). A partir dos resultados desta avaliação preliminar, as ações educativas foram direcionadas e constituíram de dinâmica de grupo, oficina de sucos saudáveis, palestra sobre alimentação e entrega de folder complementar. A avaliação nutricional indicou que a alimentação das participantes estava inadequada e não atendia às recomendações específicas para pacientes com câncer de mama. A avaliação do IMC classificou as participantes como com sobrepeso e a RCQ média do grupo ficou acima do ponto de corte estabelecido. As ações educativas proporcionaram diferentes situações para que as participantes
\end{abstract}

\begin{abstract}
This study aimed to conduct nutrition evaluation in women with breast cancer seeing a support group that offers orientation and therapeutic support. Based on the results of the evaluation, food and nutritional education (FNE) actions were developed and presented. The activities were conducted by a group of undergraduate students who attended the discipline of FNE within the Nutrition course at the Federal University of Lavras (UFLA). The quality of the participants' diet was assessed by means of a questionnaire based on the Food Guide of the Brazilian Ministry of Health. Anthropometric measurements were obtained to calculate body mass index (BMI) and waist-hip ratio (WHR). From the results of the preliminary assessment, the content of the educational activities were directed. These activities consisted in group dynamics, a workshop of healthy juices, a lecture on nutrition, and the delivery of folders containing information on healthy eating habits. The nutritional evaluation indicated that the participants' diet was inadequate and was not in agreement with specific recommendations for patients with breast cancer. BMI assessment classified participants as overweight, and mean WHR of the group was above the limit. The proposed activities provided different situations so that the participants would be able to develop critical skills so as to make better choices regarding nutrition. The undergraduate
\end{abstract}


pudessem desenvolver a capacidade crítica de fazer melhores escolhas com relação a sua alimentação. Para os estudantes de Nutrição, a experiência contribuiu para que eles, no papel de educadores, pudessem utilizar o que aprenderam com o objetivo de transformação da realidade, gerando a possibilidade de reflexão sobre as dimensões do outro, o que é fundamental no processo de formação desses profissionais.

Palavras-chave: Educação alimentar e nutricional Câncer de mama, Práticas sociais, Método intuitivo.

Área temática: Nutrição e Saúde.

Linha de extensão: Educação Alimentar e Nutricional. students who conducted the activities were able, due to the experience, to act as educators and use what they learned in order to transform reality, thus creating the possibility to reflect on the dimensions of the other, which is a key factor in the formation of these professionals.

Keywords: Food and nutrition education, Breast cancer, Social practices, Intuitive method.

\section{Introdução}

O câncer de mama é o tipo de câncer que mais acomete as mulheres em todo o mundo, tanto em países em desenvolvimento quanto em países desenvolvidos. Cerca de 1,67 milhões de casos novos dessa neoplasia foram esperados para o ano de 2012, em todo o mundo, o que representa $25 \%$ de todos os tipos de câncer diagnosticados nas mulheres. Já no Brasil, 57.120 casos novos de câncer de mama foram esperados em $2014^{1}$, o que representa $10 \%$ dos cânceres diagnosticados em toda a população, colocando a doença como um dos maiores problemas de saúde pública da atualidade.

O diagnóstico do câncer de mama, assim como todo o percurso da doença, é permeado por muitas inquietações relacionadas à morte, às mutilações e à dor, constituindo-se num período marcado por muita angústia, sofrimento e ansiedade. ${ }^{2}$ Além disso, o ganho de massa corporal dos pacientes em tratamento quimioterápico é ocorrência comum e a obesidade é considerada um fator de risco para metástase além de aumentar o desenvolvimento de tumores. ${ }^{3}$ A obesidade traz ainda repercussões de ordem metabólica e cardiovascular significativas e tem papel relevante dentro dos fatores de mal prognóstico para o câncer de mama, aumentando as taxas de mortalidade dessa população. ${ }^{4}$

Por suas características, o tratamento do câncer de mama traz ainda repercussões importantes no que se refere à identidade feminina. Além da perda da mama ou de parte desta, os tratamentos complementares podem impor a perda dos cabelos, a parada ou irregularidade da menstruação e a infertilidade, fragilizando ainda mais o 
sentimento de identidade da mulher. Isso comporta representações e significados atribuídos à doença ao longo da história e da cultura, interferindo nas relações interpessoais, principalmente nas mais íntimas e básicas da mulher. ${ }^{5}$

Sendo assim, ações que atuem sobre os determinantes do processo saúde-doença e que promovam qualidade de vida e suporte psicológico são fundamentais para essas pacientes. É nesse contexto, portanto, que se destaca o papel da educação alimentar e nutricional (EAN), que pode atuar tanto na prevenção e controle da doença como nas relações íntimas e interpessoais das pacientes.

As ações de EAN têm como requisitos básicos o desenvolvimento de abordagens educativas que permitam abraçar os problemas alimentares em sua complexidade, tanto na dimensão biológica como na social e cultural. As abordagens inter e transdisciplinares surgem assim como opções que podem oferecer caminhos alternativos. ${ }^{6}$

Isto vai ao encontro do pensamento de Pestalozzi (1746-1825), sobre o romantismo e o nascimento da pedagogia social, onde $\operatorname{Lima}^{7}$ menciona:

\footnotetext{
Se todo homem deve ser educado, independente de condição social, deve, portanto, ser aperfeiçoado em sua integralidade de coração (educação moral), mente (educação intelectual) e mão (educação profissional). Essa síntese mostra que, para Pestalozzi, não basta educar a mente ou formar a virtude. É preciso fazer as duas coisas e ainda fornecer ferramentas de trabalho.
}

Compreende-se, assim, a importância do desenvolvimento de instrumentos práticos que auxiliem na EAN como forma de promover a saúde a partir de uma melhor alimentação, bem como a necessidade de formas dinâmicas de atividades de educação nutricional para que novos conhecimentos a respeito de alimentação e nutrição sejam assimilados pela população trabalhada. ${ }^{8}$

Para os pacientes com câncer de mama, estas ações são ainda mais importantes, pois a alimentação adequada desempenha papel fundamental na prevenção da recidiva da doença e envolve, segundo recomendações do World Cancer Research Fund - American Institute for Cancer Research (WCRF-AICR) ${ }^{9}$, melhorias na qualidade da alimentação por meio da limitação do consumo de alimentos calóricos, ricos em açúcares, gordura e sal, 
carnes vermelhas e processadas e bebidas alcoólicas, bem como aumento do consumo de vegetais.

Neste contexto, visando promover ações de EAN como estratégia de formação de alunos em concomitância com práticas sociais, o presente trabalho teve como objetivo avaliar a qualidade da alimentação e obter medidas antropométricas para o cálculo do índice de massa corporal (IMC) e da relação cintura quadril (RCQ) de pacientes com câncer de mama que frequentavam um grupo de apoio para oferta de atividades de acolhimento, orientação e suporte terapêutico e, com base nestas informações, elaborar e divulgar as ações de EAN desenvolvidas para este grupo de apoio.

\section{Metodologia}

\section{Natureza da população em estudo}

A população em estudo foi constituída por 8 pacientes usuárias do sistema público de saúde, que frequentavam um grupo de apoio que realizava atividades de acolhimento, apoio, orientação e suporte terapêutico às pacientes no município de Lavras-MG. O grupo era conduzido por uma equipe multidisciplinar, composta por psicólogo, nutricionista e enfermeira que se reuniam mensalmente para realização das atividades. Os critérios de inclusão das participantes adotados foram: ser do sexo feminino, ser atendida no referido centro e estar tanto em tratamento como em fase de remissão do câncer de mama.

A intervenção nutricional educativa referiu-se ao conjunto de atividades desenvolvidas por um grupo de estudantes que cursavam a disciplina de EAN do curso de graduação em Nutrição da Universidade Federal de Lavras (UFLA). Os estudantes eram orientados pelo professor responsável pela disciplina e supervisionados pela nutricionista do local.

Inicialmente, foi apresentada uma proposta de EAN a ser aprovada pelos responsáveis do grupo de apoio. Esta proposta incluía, primeiramente, a aplicação de um questionário para avaliar a qualidade da alimentação das participantes e a obtenção de medidas antropométricas para cálculo do IMC e da RCQ. Estes resultados foram utilizados para direcionar o conteúdo a ser abordado nas ações educativas 
subsequentes, sendo elas uma dinâmica de grupo, uma oficina de sucos saudáveis e uma palestra, com posterior entrega de folder complementar da intervenção.

Após aprovação da proposta pelos responsáveis do grupo de apoio, as participantes do estudo/acompanhamento assinaram termos de consentimento livre e esclarecido, além de autorização de divulgação de imagens. No entanto, optou-se por utilizar as iniciais do nome das participantes ao apresentar seus relatos, sendo as imagens expostas apenas para o próprio grupo no final do estudo, como fator motivacional. O estudo atendeu às disposições referentes à pesquisas envolvendo seres humanos, tendo sido submetido ao Comitê de Ética em Pesquisa da UFLA e aprovado com CAAE n.: 30841614.3 .0000 .5148 .

\section{Avaliação Nutricional}

Inicialmente, um questionário baseado no Guia Alimentar para a População Brasileira $^{10}$ foi aplicado à população em estudo de forma a avaliar a qualidade da alimentação das participantes. No questionário havia 12 questões previamente selecionadas, que avaliaram de forma objetiva a quantidade média e a frequência ingerida de grupos de alimentos, bem como hábitos durante as refeições e consumo de água e bebidas alcóolicas.

Nesta etapa também foram coletados dados como idade e medidas antropométricas (peso, altura e circunferência da cintura e do quadril) para cálculo do IMC, expresso em $\mathrm{Kg} / \mathrm{m}^{2}$, e RCQ. Utilizou-se nestas medidas balança digital (Urbano, modelo PS180) e fita métrica (Sanny, 1,5 m comprimento e 0,1 cm de precisão). O IMC foi calculado pela equação proposta por Quételet ${ }^{11}$. A classificação dos níveis de obesidade foi obtida pela tabela de referência proposta pela Organização Mundial de Saúde ${ }^{12}$ com base nos valores do IMC (< 18,5 baixo peso; 18,5 - 24,9 peso normal; 25 - 29,9 sobrepeso; 30 34,9 obesidade classe I; 35 - 39,9 obesidade classe II; $\geq$ 40,0 obesidade classe III).

Após obtenção dos dados, as respostas para cada participante foram tabuladas e analisadas de forma descritiva, por análise de frequência, utilizando-se o software SPSS v. 20.0, sendo os resultados desta etapa utilizados para direcionar o conteúdo das ações educativas subsequentes. 


\section{Ações educativas}

Entre os meses de outubro e dezembro de 2014 foram realizadas as reuniões e atividades com as participantes, com duração média de 1 hora e 30 minutos, sendo cada atividade proposta conduzida em um dia específico, totalizando três encontros. $\mathrm{O}$ diferencial das atividades se deu pela ordem de realização e condução destas, em que se optou por conduzi-las conforme as ideologias do método intuitivo de Pestalozzi, em que a educação pode ser construída por meio dos sentimentos e emoções.

\section{Dinâmica de Grupo}

A dinâmica de grupo foi realizada em um ambiente propício a momentos individuais de reflexão, com o auxílio de música para relaxamento. A duração da reunião foi de aproximadamente 1 hora e 30 minutos. Técnicas de dinâmica de grupo foram empregadas, conforme metodologia proposta por Rodrigues e Boog ${ }^{13}$, para auxiliar as participantes a revelar detalhes do seu comportamento e compartilhar as descobertas com os demais membros do grupo, para que, coletivamente, pudessem analisar seus comportamentos e buscar estratégias para a mudança gradativa de práticas.

O planejamento seguiu um cronograma de atividades, elaborados previamente pela equipe executora, com base em roteiros de dinâmicas conhecidas. A atividade escolhida foi denominada "Dinâmica do Espelho", onde foi utilizado um espelho que ficou escondido dentro de uma caixa, de modo que, ao abri-la, a integrante pudesse ver seu próprio reflexo.

Um dos estudantes assumiu o papel coordenador e motivou o grupo: "Cada uma pense em alguém que lhe seja de grande significado. Uma pessoa muito importante para você, a quem gostaria de dedicar a maior atenção em todos os momentos, alguém que você ama de verdade... que merece todo seu cuidado, com quem está sintonizado permanentemente... Entre em contato com esta pessoa, com os motivos que a tornam tão amada por você, que fazem dela o grande sentido da sua vida...” 
Após estes momentos de reflexão, o coordenador continuou a narrativa: “...agora vocês vão encontrar-se aqui, frente a frente com esta pessoa que é o grande significado de sua vida”.

Em seguida, as integrantes foram orientadas a se dirigirem ao local onde estava a caixa, uma por vez. Todas deviam olhar o conteúdo e voltar silenciosamente para seu lugar, continuando a reflexão sem se comunicar com as demais. A partir daí, por intermédio do moderador e dos demais estudantes do grupo, pode-se iniciar as discussões que tiveram como principal objetivo estimular o autoconhecimento, possibilitar a interação e a autoavaliação.

\section{Oficina de sucos saudáveis}

O preparo do suco teve como propósito a interação entre as participantes, além de promover e estimular alegria durante o preparo e de educá-las quanto ao consumo de alimentos com propriedades funcionais especificas, motivando assim $\mathrm{o}$ desenvolvimento de outras preparações, com diferentes combinações de ingredientes em seus ambientes domésticos.

Para a elaboração do suco proposto, utilizou-se abacaxi, folhas de couve e água, conforme formulação descrita na Tabela 1.

\begin{tabular}{lcl}
\hline Ingredientes & Quantidade $(\mathbf{g}-\mathbf{m L})$ & Medida caseira \\
\hline Couve & 30 & $1 / 2$ folha \\
Abacaxi & 150 & 2 fatias médias \\
Água & 200 & 1 copo americano \\
\hline
\end{tabular}

Tabela 1. Receita do suco funcional proposto na Oficina de Sucos Saudáveis.

Os ingredientes utilizados foram incialmente higienizados, onde seguiu-se as seguintes etapas: lavagem com o auxílio de escova, enxague, imersão em água clorada (100 ppm de cloro ativo) por 15 minutos e um novo enxague em água corrente. Em seguida, o abacaxi foi descascado, picado em fatias e, juntamente com as folhas de couve inteiras e água potável, foi batido no liquidificador por aproximadamente 1 minuto para obtenção do suco. As porções foram então servidas e imediatamente consumidas pelas participantes. 
As participantes auxiliaram em todas as etapas de preparação, sendo as técnicas de higienização e de preparação explicadas de forma detalhada para que todas pudessem aprendê-las e aplicá-las posteriormente em suas casas.

Uma tabela com a composição nutricional e funcional do suco preparado foi montada (Tabela 2) e apresentada às participantes para que elas pudessem constatar a importância dos nutrientes e substâncias funcionais presentes. Para o cálculo da composição na porção de $250 \mathrm{~mL}$ do suco, utilizou-se como referência banco de dados de nutrientes ${ }^{14}$ e de uma tabela de substâncias funcionais. ${ }^{15}$

\begin{tabular}{|c|c|c|c|c|}
\hline $\begin{array}{l}\text { Nutriente / } \\
\text { Composto bioativo }\end{array}$ & $\begin{array}{l}\text { Abacaxi } \\
(150 \mathrm{~g})\end{array}$ & $\begin{array}{l}\text { Couve } \\
(30 \mathrm{~g})\end{array}$ & $\begin{array}{c}\text { Suco } \\
(250 \mathrm{~mL}) \\
\end{array}$ & Referência \\
\hline Energia (Kcal) & 72,00 & 8,10 & 80,10 & \multirow{17}{*}{14} \\
\hline Proteínas (g) & 1,35 & 0,87 & 2,22 & \\
\hline Lipídeos (g) & 0,15 & 0,15 & 0,30 & \\
\hline Carboidrato (g) & 18,45 & 1,29 & 19,74 & \\
\hline Fibra alimentar (g) & 1,50 & 0,93 & 2,43 & \\
\hline Cálcio (mg) & 33,00 & 39,00 & 72,00 & \\
\hline Magnésio (mg) & 27,00 & 10,50 & 37,50 & \\
\hline Manganês (mg) & 2,43 & 0,31 & 2,74 & \\
\hline Fósforo (mg) & 19,50 & 14,70 & 34,20 & \\
\hline Ferro (mg) & 0,45 & 0,15 & 0,60 & \\
\hline Sódio (mg) & $\operatorname{tr}^{*}$ & 3,30 & 3,30 & \\
\hline Potássio (mg) & 196,50 & 94,5 & 291,00 & \\
\hline Cobre (mg) & 0,17 & 0,01 & 0,17 & \\
\hline Zinco $(\mathrm{mg})$ & 0,15 & 0,06 & 0,21 & \\
\hline Tiamina (Vitamina B1) (mg) & 0,26 & 0,06 & 0,32 & \\
\hline $\begin{array}{l}\text { Riboflavina (Vitamina B2) } \\
\text { (mg) }\end{array}$ & 0,03 & 0,09 & 0,12 & \\
\hline Vitamina C (mg) & 51,90 & 29,01 & 80,91 & \\
\hline \multicolumn{5}{|l|}{ Flavonoides } \\
\hline Apigenina (mg) & $\mathrm{nd}^{* *}$ & 6,33 & 6,33 & 16 \\
\hline Miricetina (mg) & nd & 3,30 & 3,30 & 16 \\
\hline Kaempferol (mg) & nd & 0,004 & 0,004 & 17 \\
\hline Quercetina (mg) & nd & 0,003 & 0,003 & 17 \\
\hline
\end{tabular}




$\begin{array}{lcccc}\text { B-caroteno }(\mathrm{mg} / 100 \mathrm{~g}) & 0,03 & 1,75 & 1,78 & 18,19 \\ \text { Luteína }(\mathrm{mg} / \mathrm{100 \textrm {g }}) & 0,003 & 0,64 & 0,64 & 18,20\end{array}$

* traços; ${ }^{* *}$ não determinado na literatura científica os valores para a substância específica.

Tabela 2. Composição nutricional e funcional do suco de abacaxi com couve desenvolvido.

\section{Palestra}

Na palestra ministrada pelos estudantes foram abordadas questões como: alimentação saudável específica para a situação das pacientes, com base nas recomendações do WCRF-AICR $^{9}$ e do Guia Alimentar para a População Brasileira ${ }^{10}$ além de sinais e sintomas que ocorrem durante o tratamento do câncer de mama, noções sobre medidas caseiras, leitura de rótulos, dicas de como preservar os nutrientes dos alimentos e boas práticas de higienização e manipulação. A palestra também serviu como meio de apresentação dos alimentos ricos em antioxidantes, dando seguimento da motivação proposta pela oficina de alimentação saudável. Ao final, foi aberto um espaço para esclarecimento de dúvidas das participantes e obtenção de relatos sobre todas as atividades desenvolvidas no período. A duração da palestra foi de aproximadamente 2 horas, incluindo o espaço destinado à discussão e às perguntas.

\section{Folder educativo}

Ao final da palestra foi distribuído para as participantes um folder com informações importantes para a alimentação saudável, baseada na pirâmide de alimentos. No material, incluiu-se informações a respeito dos níveis da pirâmide de alimentos, em que foram apresentados os principais alimentos que compõem cada grupo, bem como as porções recomendadas para ingestão com base nas recomendações citadas anteriormente..$^{9,10} \mathrm{O}$ material também incluiu os passos para a correta seleção, higienização, acondicionamento e armazenamento de alimentos.

Por se tratar de um grupo de pacientes alfabetizadas, conforme informações fornecidas pela administração do grupo, adotou-se uma linguagem simples, com a utilização de 
imagens e ilustrações na montagem do folder, de forma que as informações fossem facilmente compreendidas por todas.

\section{Resultados e Discussão}

A análise das respostas do questionário indicou que a idade das participantes variou de 42 a 79 anos, sendo a idade média do grupo de $59 \pm 12$ anos. De forma geral, pode-se observar que a frequência de consumo de determinados grupos de alimentos estavam

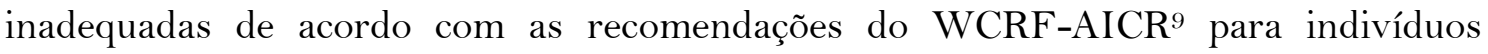
sobreviventes ao câncer e do Guia Alimentar do Ministério da Saúde ${ }^{10}$. Enquanto a minoria das participantes declararam comer quantidades adequadas de frutas, verduras, legumes, leguminosas e carboidratos, o número de porções de proteínas, frituras, embutidos, doces, balas, bolos, refrigerantes e sucos industrializados ingeridas diariamente superou as quantidades recomendadas para a maioria das participantes (Tabela 3).

\begin{tabular}{llc}
\hline Alimento & $\begin{array}{l}\text { Porções } \\
\text { recomendadas * }\end{array}$ & $\begin{array}{c}\text { Participantes } \\
\text { (\%) }\end{array}$ \\
\hline Frutas ou suco natural & $\begin{array}{l}\text { 3 ou mais porções ao } \\
\text { dia }\end{array}$ & 25,0 \\
Verduras e legumes & $\begin{array}{l}\text { 3 ou mais porções ao } \\
\text { dia }\end{array}$ & 12,5 \\
Leguminosas (feijão, lentilha, ervilha...) & $\begin{array}{l}\text { 1 porção ao dia } \\
\text { Carboidratos (arroz, farinha, macarrão...) }\end{array}$ & $\begin{array}{l}\text { 6 porções ao dia } \\
\text { 1 porção ao dia }\end{array}$ \\
$\begin{array}{ll}\text { Proteínas (carnes ou ovos) } \\
\text { Frituras, embutidos, doces, balas, bolos, } \\
\text { refrigerantes e sucos industrializados }\end{array}$ & $\begin{array}{l}\text { Máximo 1 porção ao } \\
\text { dia }\end{array}$ & 12,5 \\
${ }^{*}$ Recomendações baseadas no Ministério da Saúde & & 25,0 \\
& & 72,5 \\
\hline
\end{tabular}

Tabela 3. Porcentagem de participantes que relataram consumir porções recomendadas para cada grupo de alimentos.

Assim como legumes e verduras, as frutas são excelentes fontes de fibras, de vitaminas e minerais e de várias substâncias funcionais que contribuem para melhorar a qualidade da alimentação e prevenir a recidiva da doença. Portanto, para o público-alvo em estudo, eles devem estar presentes diariamente nas refeições, em porções adequadas. A 
maioria das participantes, no entanto, declararam que a frequência de consumo destes alimentos estava abaixo das 3 porções ou mais indicadas pelo Guia Alimentar para a População Brasileira. ${ }^{10} \mathrm{O}$ mesmo pode ser observado para as porções de carboidratos e leguminosas, em que a maioria relatou consumi-los em quantidades inferiores às recomendadas pelo guia (6 porções ao dia para carboidratos e 1 porção ao dia para leguminosas).

As fontes proteicas como carnes vermelhas, frango, peixe e ovos, por sua vez, para a maioria das participantes, estiveram presentes nas refeições em quantidades superiores às recomendadas já que $75 \%$ das participantes reportaram consumir 2 ou mais porções ao dia, enquanto o ideal seria apenas 1 porção.

Em relação à frequência de consumo de alimentos como frituras, embutidos, doces, balas, bolos, refrigerantes e sucos industrializados informada pelas participantes, esta pode ser considerada baixa, já que a maioria delas (72,5\%) reportaram consumir esses alimentos menos de 1 vez por semana ou por mês. É importante ressaltar que o consumo desses alimentos devem ser evitados ao máximo, já que eles contribuem expressivamente na elevação do consumo de sódio, gorduras e açúcares simples na dieta. ${ }^{10}$

O alto consumo de sódio e de gorduras saturadas aumenta o risco de doenças cardiovasculares, enquanto o consumo excessivo de açúcar aumenta o risco de cárie dental, de obesidade e de várias outras doenças crônicas. Além disso, evidências clínicas e epidemiológicas apontam uma relação causal indiscutível entre consumo excessivo de gorduras saturadas, sódio e açúcar com a incidência de cânceres. ${ }^{21}$ Para pacientes já diagnosticados com a doença, em fase de tratamento ou remissão, o consumo excessivo destes nutrientes é considerado ainda um dos fatores de risco para a recidiva da doença. ${ }^{9}$

Em relação às outras perguntas do questionário, relacionadas aos hábitos alimentares diários das participantes, pode-se observar que todas elas optaram por utilizar óleo vegetal para cozinhar alimentos e a maioria $(87,5 \%)$ não costumava colocar mais sal na comida que está no prato. Em relação ao consumo de água, mais de 60\% das participantes disseram consumir ao menos 8 copos de água por dia, o que pode ser 
considerada uma quantidade adequada. Quanto ao consumo de bebidas alcoólicas, 87,5\% delas afirmaram beber raramente ou nunca, o que é uma informação positiva já que o consumo frequente de bebidas alcoólicas pode ser um fator de risco para o desenvolvimento de câncer ou para a recidiva da doença. ${ }^{9,22}$

Existem evidências de que a adiposidade corporal aumentada também é fator de risco para o desenvolvimento do câncer de mama. ${ }^{9}$ Assim, excesso de gordura corporal, obesidade e circunferência da cintura elevada podem estar associadas ao aumento do risco de desenvolver a doença. Foi possível constatar neste estudo que a maioria das participantes apresentaram sobrepeso, excesso de gordura corporal e circunferência da cintura elevada.

A avaliação do IMC indicou que todas as participantes apresentaram sobrepeso, com IMC médio de $27,44 \pm 4,13 \mathrm{Kg} / \mathrm{m}^{2}$. Já a $\mathrm{RCQ}$, que é um índice utilizado para avaliar a distribuição de gordura corporal, tem como ponto de corte estabelecido para discriminar valores inadequados de RCQ para o sexo feminino de $0,8 .{ }^{23}$ Neste estudo, a RCQ médio das participantes foi de 0,9 \pm 0,07. Este índice variou de 0,78 a 0,96, sendo que $87,5 \%$ das participantes apresentaram RCQ acima do ponto de corte estabelecido.

Em pacientes com câncer de mama, o ganho de peso e a obesidade são ocorrências comuns. $\mathrm{O}$ ganho de peso usualmente varia entre 2 a $6 \mathrm{Kg}$ durante o primeiro ano do diagnóstico. Além disso, pacientes obesas com câncer de mama apresentam maior risco para metástase e propensão elevada para o desenvolvimento de tumores e recidiva bem como elevada taxa de mortalidade quando comparadas às não obesas. ${ }^{3}$

Já a obesidade centralizada na região abdominal, caracterizada neste estudo pelo alto índice de RCQ, traz repercussões de ordem metabólica e cardiovascular significativas e tem papel relevante dentro dos fatores de mal prognóstico para o câncer de mama, aumentando as taxas de mortalidade dessa população. ${ }^{4}$

Esses resultados reforçaram a necessidade de ações educativas de modo a melhorar a qualidade da dieta das participantes. Neste trabalho, estas ações focaram, principalmente, em educar quanto ao balanço das porções, inclusão de alimentos de origem vegetal ricos em compostos bioativos, com baixo teor de açúcares, gorduras e 
sódio e diminuição do consumo de carnes e derivados e alimentos calóricos além do

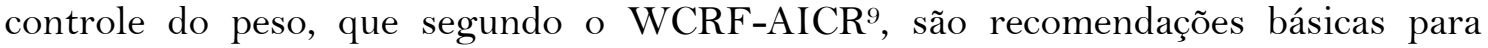
indivíduos que sobreviveram ao câncer, e que contribuem fortemente para a prevenção da recidiva da doença.

\section{Ações educativas}

A dinâmica de grupo despertou interesse nas participantes e foi canal de interação, uma vez que algumas estavam frequentando pela primeira vez a reunião do grupo. De fato, a dinâmica de grupo é uma ferramenta eficaz para auxiliar as participantes a revelar detalhes do seu comportamento alimentar e compartilhar as descobertas com os demais membros do grupo, para que, coletivamente, possam analisar seus comportamentos e buscar estratégias para a mudança gradativa de práticas. ${ }^{13}$

Muitos são os conflitos encontrados na literatura com relação aos efeitos do tratamento do câncer de mama, e vários são os instrumentos utilizados para medir a qualidade de vida; porém, de modo geral, o tratamento apresenta grande influência negativa na qualidade de vida dessas pacientes, ${ }^{5}$ o que pode ser constatado nos relatos das participantes.

No momento aberto para o debate, algumas se emocionaram e relataram um despertar para a autovalorização, uma vez que a maioria já eram mães e se mostraram preocupar muito com a família, mesmo diante da doença que enfrentam.

T.H.G: "A partir da dinâmica do espelho passei a me olhar com outros olhos, cuidando um pouco mais de mim e não só dos que estão ao meu redor."

M.I.F: “[ [... aprendi que para cuidar das pessoas que estão em minha volta, preciso antes estar bem comigo mesmo, para que isso reflita numa convivência harmoniosa e feliz com a minha família."

Durante a atividade, pode-se observar ainda grande interesse e adesão na atividade proposta. A dinâmica de grupo, portanto, foi considerada eficiente pois contribuiu para promover a integração do grupo, estimular o autoconhecimento, desenvolver o senso 
crítico, aproximar as participantes do universo alimentar, além de possibilitar a interação e a autoavaliação.

Destaca-se, nesse caso, a necessidade de as atividades de EAN contribuírem para que o sujeito seja capaz de analisar criticamente sua alimentação e a do grupo social em que está inserido e, dessa forma, decidir conscientemente sobre ela. A manutenção da saúde e da qualidade de vida é, nesse caso, consequência de uma análise crítica e emancipada do sujeito sobre sua própria alimentação e bem-estar, ${ }^{24}$ o que pode trazer muitos benefícios, principalmente para aqueles acometidos por doenças como câncer, em que a qualidade da alimentação exerce papel fundamental na cura e prevenção da recidiva da doença.

Para a oficina de sucos saudáveis, a receita escolhida foi o suco de abacaxi com couve, pois estes alimentos, conforme apresentado na Tabela 2, são fonte de vitaminas, principalmente vitamina $\mathrm{C}$, um importante antioxidante. Além disso, esses alimentos são fontes de substâncias funcionais como flavonoides e carotenoides como $\beta$-caroteno e luteína, que também proporcionam diversos benefícios à saúde como ação antioxidante e auxílio na redução de processos inflamatórios ${ }^{25}$. Rock e DemarkWahnefried, em uma revisão de evidências relacionando nutrição e sobrevida após diagnóstico de câncer de mama, enfatizam os efeitos benéficos dos compostos bioativos presentes na dieta, através das atividades antioxidantes e sobre os hormônios gonadais, na diminuição do risco de progressão do câncer de mama ${ }^{26}$, constatando-se assim os benefícios do suco preparado para este público-alvo. O abacaxi ainda apresenta em sua constituição a enzima bromelina, que contribui para uma melhora no processo digestivo. ${ }^{27}$

Durante a oficina de sucos saudáveis, percebeu-se boa integração entre estudantes e as participantes, já que laços já haviam sido estabelecidos nas etapas anteriores. Além disso, surgiu por parte das participantes outras sugestões de combinações para sucos saudáveis e associação com outras situações de suas realidades, como a alimentação dos filhos e netos, sendo a oficina, um estímulo e apoio à melhora de hábitos saudáveis, conforme pode-se perceber em alguns relatos coletados nas reuniões posteriores. 
F.G.T: "Gostei muito do suco com couve, levei a receita para casa e estou fazendo uso com a minha família toda semana.”

M.P.S: "Estou congelando o suco, pois, acho bem mais fácil quando vou prepará-lo, essa dica foi bem útil, já que não disponho de muito tempo, por conta do trabalho e afazeres do lar e como tenho netos que moram comigo, foi uma boa de incluir algo saudável na alimentação deles."

Estímulos como este são de grande importância uma vez que a ingestão de alimentos saudáveis, ricos em nutrientes e substâncias funcionais podem contribuir diretamente com a manutenção da saúde das pacientes e prevenção da recidiva da doença.

Visando melhorar o nível de conhecimento das pacientes em relação aos temas trabalhados anteriormente, bem como esclarecer eventuais dúvidas, propôs-se a ministração de uma palestra e a distribuição dos folders informativos. Na palestra, foram abordados temas relacionados à alimentação saudável específica para a situação

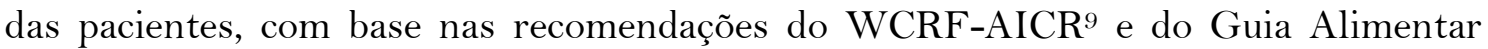
para a População Brasileira ${ }^{10}$, além de sinais e sintomas durante o tratamento do câncer de mama, noções sobre medidas caseiras, leitura de rótulos, dicas de como preservar os nutrientes dos alimentos e boas práticas de higienização e manipulação. A palestra também serviu como meio de apresentação dos alimentos ricos em antioxidantes, dando seguimento da motivação proposta pela oficina de alimentação saudável.

Levando em consideração que, normalmente, muitas deficiências ocorrem no recebimento de orientações nutricionais durante o tempo de tratamento e que o tipo de orientação oferecida às pacientes diagnosticadas com câncer de mama e frequentam o sistema público de saúde na maioria é geral e não individualizado e monitorado, ações educativas como as propostas neste trabalho podem contribuir expressivamente com a melhoria da qualidade de vida das pacientes. Segundo Tavares e $\operatorname{Trad}^{28}$, isso envolve o desenvolvimento de hábitos saudáveis relacionados à alimentação e a atividades físicas semanais, assim como melhoria nas relações sociais, que foram pontos extensivamente trabalhados nas intervenções propostas. 
Sendo assim, atividades que trabalhem conteúdos alimentares e busquem inseri-los na realidade dos indivíduos podem promover mudanças de conhecimento e de práticas alimentares. A educação que promove construção de conhecimento promove mudança. ${ }^{29}$ Assim, consegue-se melhorar hábitos alimentares seja de adultos, crianças ou de públicos-alvo com requisitos específicos, como os deste estudo.

\section{Reflexão das metodologias de intervenções educativas adotadas}

O nutricionista, no exercício do seu papel de educador em ações de educação nutricional, deve utilizar o seu saber com o objetivo de transformação da realidade. Para tanto, esse profissional deve estar atento em respeitar o tempo e as habilidades do outro, as regras da boa convivência com as diferenças, deve parar para escutar e para olhar, parar para sentir e perceber detalhes, não agir com automatismo e ter paciência, permitindo o tempo e o espaço para a diferença do outro. ${ }^{24}$

Esses pensamentos podem ser associados aos de Jean-Jacques Rousseau (1712 - 1778) na concepção do Emílio ${ }^{30}$, na qual o pensador concebe os ideais da formação humana. Paiva $^{31}$ cita que estes ideais, quando relacionados aos objetivos e às finalidades do ato educativo, nos faz repensar sobre o processo de construção do conhecimento não como atos formais e mecânicos que devem ser levados a termo por uma exigência institucional. Evidenciar a dimensão natural do ser humano no processo de aprendizagem e ensino é o desafio e, ao mesmo tempo, o estímulo que se coloca aos processos educativos dos nossos dias.

Toda essa temática foi extensivamente trabalhada neste trabalho, por meio das ações de EAN desenvolvidas com as participantes. Tanto do ponto de vista nutricional, psicológico e social, estas ações estimularam o grupo a adotar melhores escolhas em relação a sua alimentação, visto que a importância delas para prevenção da recidiva da doença foi bastante enfatizada. Além disso, pôde-se constatar, por meio dos relatos das participantes, a importância que os métodos adotados, construídos através dos sentimentos e emoções, desempenharam sobre o dia a dia das participantes.

Em concomitância a esses processos, os alunos puderam aprender de forma construtiva e intuitiva a forma de aplicar os recursos e ajudar efetivamente na formação do ser 
humano, não só diante às visões técnicas da nutrição, mas sim no somatório de vários contextos que englobam a multidisciplinaridade do curso de graduação em Nutrição.

\section{Conclusão}

A avaliação nutricional preliminar às ações educativas indicaram que a alimentação das participantes estava inadequada e não atendia às recomendações específicas para pacientes com câncer de mama. A avaliação do IMC classificou as participantes como com sobrepeso e a RCQ média do grupo ficou acima do ponto de corte estabelecido.

As ações educativas propostas neste trabalho proporcionaram diferentes situações para que as participantes pudessem desenvolver a capacidade crítica de fazer melhores escolhas com relação a sua alimentação. Por intermédio de estratégias que superaram a mera transmissão de informações, estes recursos visaram a construção de conhecimento sobre alimentação saudável e a conscientização dos pacientes sobre a importância de uma alimentação adequada para uma boa recuperação e prevenção da recidiva da doença.

Para os estudantes de Nutrição que conduziram as atividades, a experiência contribuiu para que eles, no papel de educadores, pudessem utilizar aquilo que aprenderam, com o objetivo de transformação da realidade, gerando a possibilidade de reflexão sobre as dimensões do outro, o que é fundamental no processo de formação desses profissionais.

\section{Contribuições individuais dos autores}

Bruna Nogueira Andrade: planejamento e execução das atividades práticas, aquisição de dados.

Edmar Ramos: planejamento e execução das atividades práticas, aquisição de dados.

Geisa Oliveira Rocha: planejamento e execução das atividades práticas, aquisição de dados.

Rafaela Corrêa Pereira: tabulação, análise e interpretação de dados, redação e revisão do artigo. 
Michel Cardoso de Angelis-Pereira: orientação no planejamento e execução das atividades práticas, redação e revisão do artigo.

Glaucia Imaculada Soares Pereira: supervisão das atividades práticas, aquisição de dados.

Maria Camila Lima: aquisição de dados.

\section{Referências}

1. INCA, Instituto Nacional de Câncer. Estimativa 2014: Incidência de câncer no Brasil. Instituto Nacional de Câncer José Alencar Gomes da Silva, Coordenação de Prevenção e Vigilância. Rio de Janeiro: INCA, 2014, 124 p.

2. FERREIRA, Simone Mara de Araújo; PANOBIANCO, Marislei Sanches; GOZZO, Thaís de Oliveira; ALMEIDA, Ana Maria de. A sexualidade da mulher com câncer de mama: Analise da produção cientifica de enfermagem. Texto \& Contexto - Enfermagem, Florianópolis, v.22, n.3, p.835-42, 2013.

3. FIGUEIREDO, Ana Cláudia Dias Sousa; SAÇO, Lívia Fabiana; DAMASCENO, Vinícius Oliveira; FERREIRA, Roberta Nogueira Furtado; FERREIRA, Eliana Lúcia. Associação entre variáveis antropométricas e o tratamento para o câncer de mama. ConsScientiae Saúde, v. 13, n. 1, p. 93-100, 2014.

4. THIVAT, Emilie; THERONDEL, Sophie; LAPIROT, Olivier; ABRIAL, Catherine; GIMBERGUES, Pierre; GADEA, Emilie; PLANCHAT, Elö̈se; KWIATKOWSKI, Fabrice; MOURET-REYNIER, Marie A.; CHOLlET, Philippe; DURANDO, Xavier Weight change during chemotherapy changes the prognosis in non metastatic breast cancer for the worse. BMC Cancer, v.10, p. 648, 2010.

5. SILVA, Elaine Cristina de Souza da; SILVA, Josiane Maria da; SILVA, Letícia Ferreira da; BATISTA, Roberta Firmino; SAMPAIO, Shirlene; CARNEIRO, Paula Frassinetti Pereira. Câncer de mama, menopausa e qualidade de vida. Revista Brasileira de Ginecologia e Obstetrícia, v. 1, n. 3, p. 85-93, 2014.

6. BOOG, Maria Cristina Faber; VIEIRA, Carla Maria; OLIVEIRA, Nayara Lúcia; FONSECA, Odila; L'ABBATE, Solange. Utilização de vídeo como estratégia de educação nutricional para adolescentes: "Comer... o fruto ou o produto?" Revista de Nutrição, v.16, n. 3, p. 281-293, 2003.

7. LIMA, João Francisco Lopes de. Pestalozzi: o Romantismo e o nascimento da Pedagogia Social. Ciência e Letras, Porto Alegre, n.47, p. 123-115, 2010.

8. MENDES-NETTO, Raquel Simões; Teixeira, Pryscila Dryelle Sousa; REIS, Bruna Zavarize; VIEIRA, Diva Aliete; COSTA, Dayanne da; COSTA, Jamille Oliveira. Ações educativas para promoção de hábitos alimentares saudáveis: relato 
de uma experiência. Revista de Extensão Universitária da UFS, São Cristovão, n. 2, pp.191-199, 2013.

9. WCRF, AICR. Food, nutrition, physical activity, and the prevention of cancer: a global perspective. Washington, DC: WCRF, World Cancer Research Fund; AICR, American Institute for Cancer Research; 2007.

10. BRASIL. Ministério da Saúde. Coordenação Geral de Alimentação e Nutrição (CGAN). Política Nacional de Alimentação e Nutrição. Como está sua alimentação? Disponível em: http://nutricao.saude.gov.br/teste_alimentacao.php. Acesso em 07 mar 2015.

11. QUÉTELET, A. Antropométrie ou mesure des différentes facultés de l'homme. Bruxelles, C. Muquardt, 1870.

12. World Health Organization technical report series. Obesity: preventing and managing the global epidemic. Report of a WHO consultation, 2000.

13. RODRIGUES, Érika Marafon; BOOG, Maria Cristina Faber. Problematização como estratégia de educação nutricional com adolescentes obesos. Cadernos de Saúde Pública, v.22, n.5, p.923-931, 2006.

14. TACO. Tabela Brasileira de Composição de Alimentos. NEPA - Unicamp. 4 ed. Campinas: NEPA - UNICAMP, 2011. 161p.

15. PEREIRA, Rafaela Corrêa; ANGELIS-PEREIRA, Michel Cardoso de. Compostos fenólicos na saúde humana: do alimento ao organismo. Lavras: Editora UFLA, 2014. 90p.

16. CHU, Yan-Hwa; CHANG, Chao-Lin, HSU, Hsia-Fen. Flavonoid contente of several vegetables and their antioxidante activity. Jounal of the Science of Food and Agriculture, v. 80, p. 561-566, 2000.

17. HERTOG, Michael G. L.; HOLLMAN, Peter C. H., KATAN, Martijn B. Content of potentially anticarcinogenic flavonoids of 28 vegetables and 9 fruits commonly consumed in The Netherlands. Journal of Agriculture and Food Chemistry, v. 40, p. 2370-2383, 1992.

18. HEINONEN, Marina I.; OLLILAINEN, Velimatti; LINKOLA, Eeva K.; VARO, Pertti T.; KPOIVISTOINEN, Pekka E. Carotenoids in Finnish foods: vegetables, fruits and berries. Journal of Agriculture and Food Chemistry, v. 37, p. 655659, 1989.

19. NACTIGALL, Aline Manke; STRINGHETA, Paulo Cesar; FIDELIS, Priscilla Cardoso, NACHTIGALL, Fabiane Manke. Determinação do teor de luteína em hortaliças. B. CEPPA, v. 25, n. 2, p. 181-192, 2007.

20. DELlA LUCIA, Ceres Mattos; CAMPOS, Flávia Milagres; MATA, Gardênia Márcia Silva Campos; SANT’ANA, Helena Maria Pinheiro. Controle de perdas de carotenóides em hortaliças preparadas em unidade de alimentação e nutrição hospitalar. Ciência e Saúde Coletiva, v. 13, n. 5, p. 1627-1636, 2008. 
21. MONTEIRO, Carlos Augusto; MONDINI, Lenise; COSTA, Renata B. L. Mudanças na composição e adequação nutricional da dieta familiar nas áreas metropolitanas do Brasil (1988-1996). Revista de Saúde Pública, v. 34, n. 3, p. 251-58, 2000.

22. LAUTER, Dagmar Scholl; BERLEZI, Evelise Moraes; ROSANELLI, Cleci de Lourdes Schmidt Piovesan; LORO, Marli Maria; KOLANKIEWICZ, Adriane Cristina Bernat. Câncer de mama: estudo caso controle no Sul do Brasil. Revista Ciências e Saúde, v. 7, n. 1, p. 19-26, 2014.

23. MACHADO, Paula Aballo Nunes.; SICHIERI, Rosely. Relação cintura-quadril e fatores de dieta em adultos. Revista de Saúde Pública, v. 36, n. 2, p. 198-204, 2002.

24. REZENDE, Eliane Garcia; MURTA, Nadja Maria Gomes; MACHADO, Virgínia Campos. Educação Nutricional e a cultura como questão. Ponto-e-Vírgula, v. 10, p.89-100, 2011.

25. GAMMONE, Maria Alessandra; RICCIONI, Graziano; D’ORAZIO, Nicolantonio. Carotenoids: potential allies of cardiovascular health? Food and Nutrition Research, v. 59, p. 1-11, 2015.

26. ROCK, Cheryl; DEMARK-WAHNEFRIED, Wendy. Nutrition and survival after the diagnosis of breast cancer: a review of the evidence. Journal of Clinical Oncology, v. 20, n. 15, p. 3302-3316, 2002.

27. GRANADA, Graziele Guimarães; ZAMBIAZI, Rui Carlos; MENDONÇA, Carla Rosane Barboza. Abacaxi: produção, mercado e subprodutos. B. CEPPA, v.22, n.2, p. 405-422, 2004.

28. Tavares e Trad

29. VERDE, Sara Maria Moreira Lima; OLINDA, Querubina Bringel. Educação nutricional: uma ferramenta para alimentação saudável. Revista Brasileira em Promoção da Saúde, v. 23, n. 3, p. 197-198, 2010.

30. ROUSSEAU, Jean-Jacques. Emílio ou da educação. 2 ed. São Paulo: Difusão Europeia do Livro, 1973. 744p.

31. PAIVA, Wilson Alves. A formação do homem em Emílio de Rousseau. Educação e Pesquisa, São Paulo, v.33, n.2, p. 323-333, 2007. 